\title{
Prone ventilation as treatment of acute respiratory distress syndrome related to COVID-19
}

\author{
Patrizio Petrone ${ }^{1}$ (C) Collin E. M. Brathwaite ${ }^{1} \cdot$ D'Andrea K. Joseph $^{1}$
}

Received: 9 July 2020 / Accepted: 31 October 2020 / Published online: 17 November 2020

(c) Springer-Verlag GmbH Germany, part of Springer Nature 2020

\begin{abstract}
Prone ventilation refers to the delivery of mechanical ventilation with the patient lying in the prone position. The improvement of oxygenation during prone ventilation is multifactorial, but occurs mainly by reducing lung compression and improving lung perfusion. CT imaging modeling data demonstrated that the asymmetry of lung shape leads to a greater induced pleural pressure gravity gradient when supine as compared to prone positioning. Although proning is indicated in patients with severe ARDS who are not responding to other ventilator modalities, this technique has moved away from a salvage therapy for refractory hypoxemia to an upfront lung-protective strategy intended to improve survival in severe ARDS, especially due to the current COVID-19 pandemic. In view of different roles, we surgeons had to take during the COVID-19 pandemic, it is of importance to learn how to implement this therapeutic measure, especially in a surgical critical care unit setting. As such, this article aims to review the physiological principles and effects of the prone ventilation, positioning, as well as its contraindications and complications.
\end{abstract}

Keywords Prone ventilation · Acute respiratory distress syndrome $\cdot$ COVID-19

\section{Introduction}

Prone ventilation refers to the delivery of mechanical ventilation with the patient lying in the prone position [1]. It may be used for the treatment of acute respiratory distress syndrome (ARDS) as a strategy to improve oxygenation and was first proposed in the 1970s as a method to improve gas exchange in ARDS. To reduce atelectasis in injured lungs, Bryan [2] proposed prone positioning, theorizing that it would reduce pleural pressure gradients and restore aeration to dorsal lung segments. Clinical case series supported this concept, documenting significant improvement in oxygenation with prone positioning [3]. Subsequent studies suggested that prone positioning improves oxygenation in most patients (70-80\%) with ARDS [4-6]. Prone positioning was then established as a rescue strategy for severe hypoxemia.

Patrizio Petrone

patrizio.petrone@nyulangone.org

1 Department of Surgery, NYU Long Island School of Medicine, NYU Langone Hospital-Long Island, 222 Station Plaza N., Suite 300, Mineola, NY 11501, USA
Subsequent observations of improvement in oxygenation with simple patient rotation dominated the next several decades of research. Despite evidence in favor of prone ventilation, adoption of this strategy has been slow in the United States compared with Europe, likely due to the perceived operational barriers to performing it.

\section{Physiological principles and effects}

The improvement of oxygenation during prone ventilation is multifactorial, but occurs mainly by reducing lung compression and improving lung perfusion. Changes in the distribution of extravascular lung fluid and secretions may also play a role. Prone positioning reduces the difference between the dorsal and ventral transpulmonary pressure, making ventilation more homogeneous [7], and leading to a decrease in ventral alveolar overinflation and dorsal alveolar collapse [8]. As a result, there is reduced alveolar distension limiting ventilator-associated lung injury, and allowing for opening of alveoli that had collapsed during supine ventilation. The outcome is an improvement in ventilation and oxygenation [8-11]. Multiple physiological studies support the theory that placing a person in the prone position promotes more homogeneous 
aeration of the lung in ARDS. CT imaging modeling data demonstrated that the asymmetry of lung shape leads to a greater induced pleural pressure gravity gradient when supine is compared to prone positioning [12]. The ability to attenuate mechanical lung injury may be more beneficial clinically. Lung compression by both the heart and the diaphragm, especially in obese patients and when the abdomen is left unsupported, can be reduced by prone positioning, improving ventilation and oxygenation as well [13]. Moreover, improved perfusion of portions of the lung is thought to be partially responsible for the enhanced oxygenation seen with prone ventilation. The lung continues to receive the majority of the blood flow as alveoli reopen. Increases in cardiac outputs have been observed and thought to be the effect of increased lung recruitment and reduction in pulmonary vasoconstriction, resulting in increases in preload and decreased afterload and pulmonary vascular resistance [7, 14-16]. It was previously hypothesized that prone ventilation permits the redistribution of blood flow based on gravitational gradient, but there is little evidence to support this.

Gattinoni et al. [17] suggested two type of pneumonia in COVID-19. Type 2 is the typical low compliance pneumonia with bilateral dense infiltrates that should be managed like ARDS and where proning can be used in severe ARDS according to guidelines. Type 1 pneumonia, on the other hand, is a high compliance pneumonia with scanty opacities. In this type of pneumonia, proning may be used as a rescue measure for redistribution of pulmonary blood flow rather than opening up collapsed lung. However, when applied to COVID-19 pneumonia, it has been found that strict H (High elastance) and L (Low elastance) groups are uncommon and the disease manifests as a spectrum with intermediate findings also [18]. Non-COVID studies and even non-infectious etiologies have suggested the role of awake proning in improving oxygenation in ARDS patients due to infectious reasons [19-22]. Moreover, a study by Pelosi et al. [23] demonstrated improved oxygenation and functional residual capacity in healthy lungs even in the absence of disease.

Although proning is indicated in patients with severe ARDS who are not responding to other ventilator modalities, this technique has moved away from a salvage therapy for refractory hypoxemia to an upfront lung-protective strategy intended to improve survival in severe ARDS, especially due to the current COVID-19 pandemic. Indeed, prone positioning has never been proven to have a survival benefit when used as a late rescue therapy for refractory hypoxemia. There are even reports in the literature about the application of conscious proning in awake, non-intubated, and hypoxemic Emergency Department (ED) COVID-19 patients [24], and in early self-proning in patients having hypoxemia related to COVID-19 on arrival to the ED [18]. However, prone ventilation is postulated as a rescue therapy in this population based on a randomized trial that showed a mortality benefit (PROSEVA) [6].

In a study from United Kingdom, Hallifax et al. [25] concluded that data from their cohort of patients managed on a respiratory high-dependency unit providing continuous positive airways pressure and respiratory physiotherapy support to enable awake proning show an association with successful awake proning and improved outcomes in patients receiving non-invasive respiratory support.

Winearls et al. [26] reported that the improvement in respiratory parameters achieved by prone positioning on continuous positive airways pressure provides a potential alternative to increasing the positive end-expiratory pressure, which may be both poorly tolerated in conscious patients and may exacerbate the lung injury through barotrauma. Munshi et al. [27] reported evidence that shows the effects of early, prolonged prone positioning in invasively ventilated patients with ARDS.

\section{Positioning}

In patients with severe ARDS, prone positioning is initiated after a period of $12-48 \mathrm{~h}$. Timely initiation of prone ventilation was most effective as collapsed lung units are likely to be recruited most easily during the acute phase of ARDS $[4,6]$.

Interestingly, there is no standard method for moving a patient from the supine to the prone position. It is recommended that facilities be educated in its use as moving the patient into the prone position is labor intensive [28]. Currently, our institution has implemented self-proning ventilation in awake and non-intubated COVID-19 patients (Fig. 1), and has instituted dedicated teams to prone intubated patients with severe ARDS due to COVID-19 (Figs. 2, 3).

The delivery of invasive mechanical ventilation in the prone position is similar to that employed when the patient is supine. Proning does not require additional monitoring, although the need for endotracheal suctioning should be assessed with increased frequency after the patient is placed prone as large quantities of pulmonary secretions may be produced. Electrocardiographic (EKG) leads should be placed on the back. Enteral feeds can be resumed, but care must be taken as this can be complicated by emesis and increased residual gastric volumes [29, 30]. In a study [31] using a protocol of continuous feeding and rate increased by $25 \mathrm{cc}$ every 6 hours, 25 degree head elevation and prophylactic $250 \mathrm{mg}$ of erythromycin IV every 6 hours, faster nutrition goals were achieved without increased gastric residuals, vomiting, or ventilator-associated pneumonia. Therefore, to facilitate gastric emptying, some centers closely monitor gastric residuals, adjust pharmacotherapy, and place the bed in a reverse Trendelenburg position while the patient is prone [32]. Tube feeds should be temporarily off and 


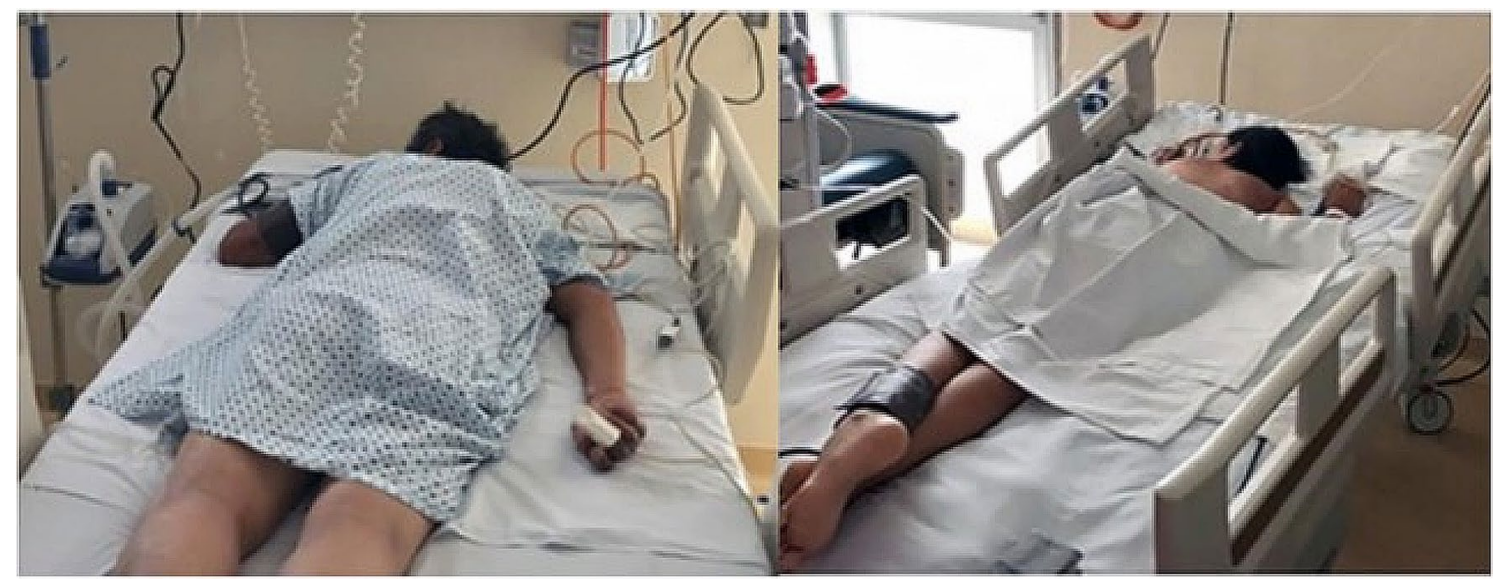

Fig. 1 Self-proning in an awake, non-intubated COVID-19 patient

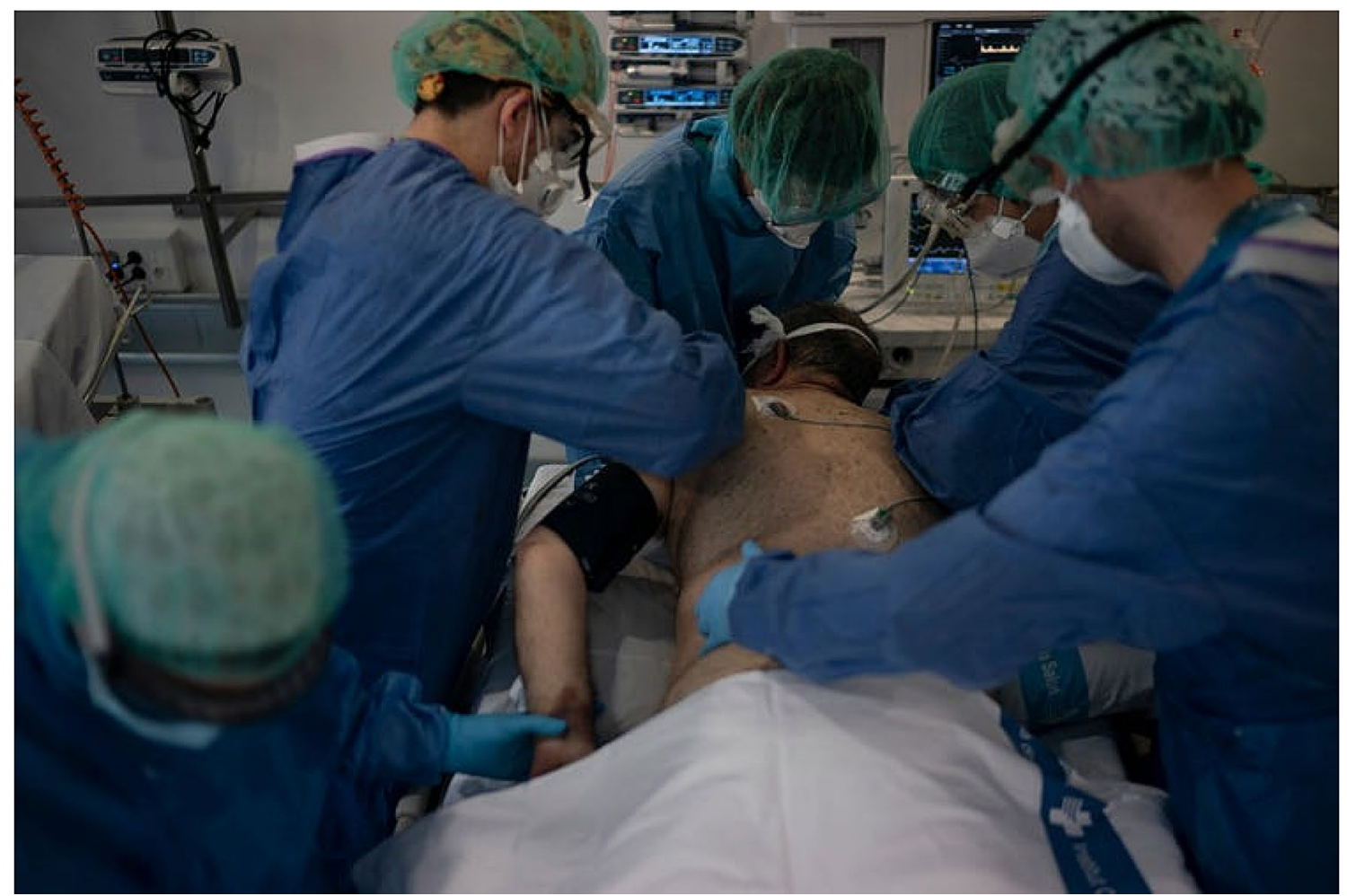

Fig. 2 NYU Langone Hospital—Long Island Prone Team in action

the stomach emptied when putting the patient back to the supine position. All patients placed in prone position require increased sedation, and most procedures and planned transport must be performed in the supine position.

The optimal duration of prone positioning is still unknown. Most studies have used either repeated sessions of prone ventilation lasting 6-8 eight hours per day $[5,33]$ or prolonged prone ventilation lasting $17-20 \mathrm{~h}$ per day $[4,6,11,34]$. Both these methods demonstrate similar results. In the PROSEVA study [6] the mean duration of time in the prone position was $17 \mathrm{~h}$ per day with an average of four sessions in total per patient. Cessation of proning is indicated when there are signs of improved oxygenation or in case acute emergencies, prolonged interventions, or surgical procedures are needed. 


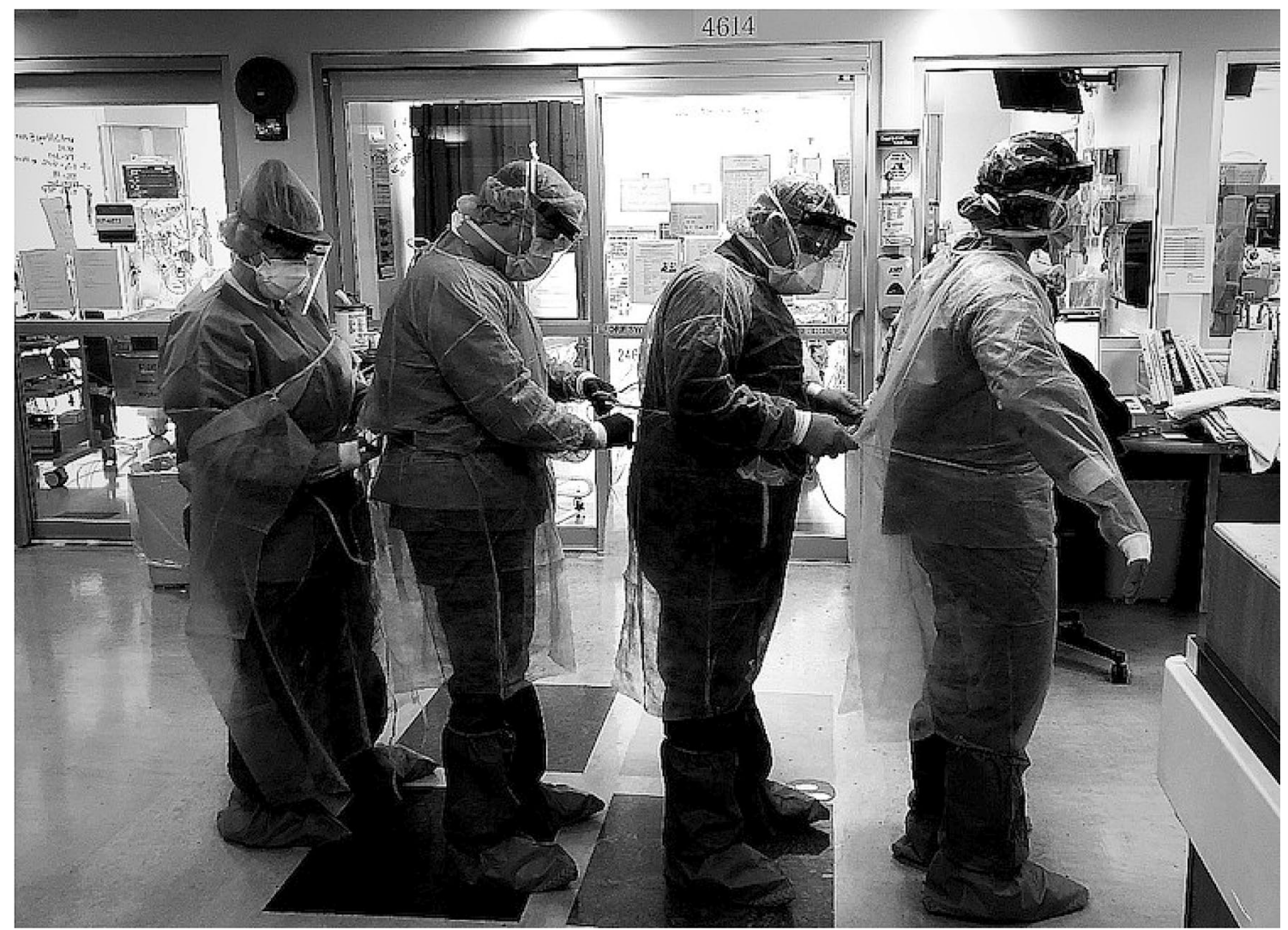

Fig. 3 Members of the NYU Langone Hospital—Long Island Prone Team help each other with their PPE

\section{Contraindications}

Absolute contraindications [35] to prone ventilation include spinal instability or at risk of spinal instability (rheumatoid arthritis), unstable fractures (especially facial and pelvic), anterior surface burns, chest tubes, open wounds, shock, pregnancy, recent tracheal surgery, and raised intracranial pressure, while relative contraindications [36] include thoracic and abdominal surgeries, difficult airway, and massive hemoptysis.

\section{Complications}

Prone positioning is not without risks. Certain complications occur, including facial and ocular edema due to venous stasis; brachial plexus neuropathy because of arm extension; and pressure ulcers, although the frequency seems to be the same as in supine position and this is mostly related to time spent prone, and occurs at different locations such as shoulder, face, chest, and knee [37]. Given the importance of pressure ulcer prevention in this type of patients, adopting a focused prevention strategy, including skin assessment and care, offloading and pressure redistribution, and dressings for prevention may contribute to a reduction in the incidence and prevalence of these largely preventable wounds. Dressings such as hydrocolloids, transparent film, and silicone may be of benefit in decreasing facial skin breakdown [38].

Additional complications include elevated intra-abdominal pressure; dislodgement of tubes and lines, including endotracheal and chest tubes and vascular catheters; increased gastric residuals and vomiting; and retinal damage, although this is unclear if relates to prone position or to severe hypoxemia [39].

\section{Final considerations and summary}

Patients with severe ARDS due to COVID-19 are candidates for prone position. It should be started early, ideally within 36-48 $\mathrm{h}$ and maintained for 18-20 consecutive hours. The best outcomes are reported when used in combination with low tidal volume and neuromuscular blockade. Positioning of the patient requires 3-5 people and with special attention being paid to placement of tubes and lines like the endotracheal tube (ETT) and central lines. Patients are prepared by preoxygenation, gastric emptying, and removal of secretions by suction of ETT and oral cavity. The EKG leads are removed and reattached to the patient's back. Pressure 
points like face, shoulder, and anterior pelvis are given additional support and repositioned frequently. Besides the listed contraindications, the least recommended patients to place in prone position are those with recent sternotomy and the ones at risk of requiring CPR or defibrillation. In conclusion, prone positioning should be considered early in the course of ARDS due to COVID-19 and be utilized until there is an improvement in gas exchange, mechanics, and overall clinical course.

Acknowledgements Dr. Patricia Cherasard, for her courtesy photographs.

Author contributions Design: PP, CEMB, and DKJ. Drafting of the manuscript: PP, CEMB, and DKJ. Critical revision: PP, CEMB, and DKJ.

\section{Compliance with ethical standards}

Conflict of interest Patrizio Petrone, Collin E.M. Brathwaite, and D'Andrea K. Joseph declare that they have not conflict of interest.

Informed consent This study is a literature review, and as such, there is no need for an informed consent.

\section{References}

1. Scholten EL, Beitler JR, Prisk GK, Malhotra A. Treatment of ARDS with prone positioning. Chest. 2017;151(1):215-24. https ://doi.org/10.1016/j.chest.2016.06.032.

2. Bryan AC, Conference on the scientific basis of respiratory therapy. Pulmonary physiotherapy in the pediatric age group. Comments of a devil's advocate. Am Rev Respir Dis. 1974;110(62):143-4. https://doi.org/10.1164/ arrd.1974.110.6P2.143.

3. Piehl MA, Brown RS. Use of extreme position changes in acute respiratory failure. Crit Care Med. 1976;4(1):13-4. https://doi. org/10.1097/00003246-197601000-00003.

4. Mancebo J, Fernández R, Blanch L, et al. A multicenter trial of prolonged prone ventilation in severe acute respiratory distress syndrome. Am J Respir Crit Care Med. 2006;173(11):1233-9. https://doi.org/10.1164/rccm.200503-353OC.

5. Guerin C, Gaillard S, Lemasson S, et al. Effects of systematic prone positioning in hypoxemic acute respiratory failure: a randomized controlled trial. JAMA. 2004;292(19):2379-87. https:// doi.org/10.1001/jama.292.19.2379.

6. Guérin C, Reignier J, Richard JC, et al. Prone positioning in severe acute respiratory distress syndrome. N Engl J Med. 2013;368(23):2159-68. https://doi.org/10.1056/NEJMoa1214103.

7. Lai-Fook SJ, Rodarte JR. Pleural pressure distribution and its relationship to lung volume and interstitial pressure. J Appl Physiol. 1991;70(3):967-78. https://doi.org/10.1152/jappl.1991.70.3.967.

8. Cornejo RA, Díaz JC, Tobar EA, et al. Effects of prone positioning on lung protection in patients with acute respiratory distress syndrome. Am J Respir Crit Care Med. 2013;188(4):440-8. https ://doi.org/10.1164/rccm.201207-1279OC.

9. Douglas WW, Rehder K, Beynen FM, et al. Improved oxygenation in patients with acute respiratory failure: the prone position. Am Rev Respir Dis. 1977;115(4):559-66. https://doi.org/10.1164/ arrd.1977.115.4.559.
10. Chatte G, Sab JM, Dubois JM, et al. Prone position in mechanically ventilated patients with severe acute respiratory failure. Am J Respir Crit Care Med. 1997;155(2):473-8. https://doi. org/10.1164/ajrccm.155.2.9032181.

11. Fridrich P, Krafft P, Hochleuthner H, Mauritz W. The effects of long-term prone positioning in patients with trauma-induced adult respiratory distress syndrome. Anesth Analg. 1996;83(6):120611. https://doi.org/10.1097/00000539-199612000-00013.

12. Tawhai MH, Nash MP, Lin C-L, Hoffman EA. Supine and prone differences in regional lung density and pleural pressure gradients in the human lung with constant shape. J Appl Physiol. 2009;107(3):912-20. https://doi.org/10.1152/japplphysiol.00324 2009.

13. Pelosi P, Croci M, Calappi E, et al. Prone positioning improves pulmonary function in obese patients during general anesthesia. Anesth Analg. 1996;83(3):578-83. https://doi.org/10.1097/00000 539-199609000-00025.

14. Albert RK, Hubmayr RD. The prone position eliminates compression of the lungs by the heart. Am J Respir Crit Care Med. 2000;161(5):1660-5. https://doi.org/10.1164/ajrccm.161.5.99010 37.

15. Jozwiak M, Teboul JL, Anguel N, et al. Beneficial hemodynamic effects of prone positioning in patients with acute respiratory distress syndrome. Am J Respir Crit Care Med. 2013;188(12):142833. https://doi.org/10.1164/rccm.201303-0593OC.

16. Nyrén $S$, Radell $P$, Lindahl $S G$, et al. Lung ventilation and perfusion in prone and supine postures with reference to anesthetized and mechanically ventilated healthy volunteers. Anesthesiology. 2010;112(3):682-7. https://doi.org/10.1097/ALN.0b013e3181 cf $40 \mathrm{c} 8$.

17. Gattinoni L, Chiumello D, Rossi S. COVID-19 pneumonia: ARDS or not? Crit Care. 2020;24(1):154. https://doi.org/10.1186/s1305 4-020-02880-z.

18. Bos LD, Paulus F, Vlaar APJ, Beenen LFM, Schultz MJ. Subphenotyping ARDS in COVID-19 patients: consequences for ventilator management. Ann Am Thorac Soc. 2020;17(9):1161-3. https ://doi.org/10.1513/AnnalsATS.202004-376RL.

19. Pérez-Nieto OR, Guerrero-Gutiérrez MA, Deloya-Tomas E, Namendys-Silva SA. Prone positioning combined with highflow nasal cannula in severe non-infectious ARDS. Crit Care. 2020;24(1):114. https://doi.org/10.1186/s13054-020-2821-y.

20. Bellone A, Basile A. Prone positioning in severe acute hypoxemic respiratory failure in the emergency ward. Emerg Care J. 2018;14(1):22-3. https://doi.org/10.4081/ecj.2018.7524.

21. Ding L, Wang L, Ma W, He H. Efficacy and safety of early prone positioning combined with HFNC or NIV in moderate to severe ARDS: a multi-center prospective cohort study. Crit Care. 2020;24(1):28. https://doi.org/10.1186/s13054-020-2738-5.

22. Scaravilli V, Grasselli G, Castagna L, Zanella A, et al. Prone positioning improves oxygenation in spontaneously breathing non intubated patients with hypoxemic acute respiratory failure: a retrospective study. J Crit Care. 2015;30(6):1390-4. https://doi. org/10.1016/j.jcrc.2015.07.008.

23. Pelosi P, Caironi P, Taccone P, Brazzi L. Pathophysiology of prone positioning in the healthy lung and in ALI/ARDS. Minerva Anestesiol. 2001;67(4):238-47.

24. Jiang LG, LeBaron J, Bodnar D. Conscious proning: an introduction of a proning protocol for non-intubated, awake, hypoxic emergency department COVID-19 patients. Acad Emerg Med. 2020. https://doi.org/10.1111/acem.14035.

25. Hallifax RJ, Porter BM, Elder PJ, Evans SB, Oxford Respiratory Group, et al. Successful awake proning is associated with improved clinical outcomes in patients with COVID-19: singlecentre high-dependency unit experience. BMJ Open Respir Res. 2020;7(1):e000678. https://doi.org/10.1136/bmjresp-2020-00067 8. 
26. Winearls S, Swingwood EL, Hardaker CL, Smith AM, et al. Early conscious prone positioning in patients with COVID-19 receiving continuous positive airway pressure: a retrospective analysis. BMJ Open Respir Res. 2020;7(1):e000711. https://doi.org/10.1136/ bmjresp-2020-000711.

27. Munshi L, Del Sorbo L, Adhikari NKJ, Hodgson CL, et al. Prone position for acute respiratory distress syndrome. A systematic review and meta- analysis. Ann Am Thorac Soc. 2017;14(S4):S280-8. https://doi.org/10.1513/AnnalsATS.20170 4-343OT.

28. Caputo ND, Strayer RJ, Levitan R. Early self-proning in awake, non-intubated patients in the emergency department: a single ED's experience during the COVID-19 pandemic. Acad Emerg Med. 2020;27(5):375-8. https://doi.org/10.1111/acem.13994.

29. Messerole E, Peine P, Wittkopp S, et al. The pragmatics of prone positioning. Am J Respir Crit Care Med. 2002;165(10):1359-63. https://doi.org/10.1164/rccm.2107005.

30. Reignier J, Thenoz-Jost N, Fiancette M, et al. Early enteral nutrition in mechanically ventilated patients in the prone position. Crit Care Med. 2004;32(4):94-9. https://doi.org/10.1097/01. CCM.0000104208.23542.A8.

31. Saez de la Fuente I, Saez de la Fuente J, Quintana Estelles $\mathrm{MD}$, et al. Enteral nutrition in patients receiving mechanical ventilation in a prone position. JPEN J Parenter Enteral Nutr. 2016;40(2):250-5. https://doi.org/10.1177/0148607114553232.

32. Reignier J, Dimet J, Martin-Lefevre L, et al. Before-after study of a standardized ICU protocol for early enteral feeding in patients turned in the prone position. Clin Nutr. 2010;29(2):210-6. https ://doi.org/10.1016/j.clnu.2009.08.004.
33. Guérin C. Prone ventilation in acute respiratory distress syndrome. Eur Respir Rev. 2014;23(132):249-57. https://doi. org/10.1183/09059180.00001114.

34. Gattinoni L, Tognoni G, Pesenti A, et al. Effect of prone positioning on the survival of patients with acute respiratory failure. $\mathrm{N}$ Engl J Med. 2001;345(8):568-73. https://doi.org/10.1056/NEJMo a010043.

35. Taccone $\mathrm{P}$, Pesenti $\mathrm{A}$, Latini R, et al. Prone positioning in patients with moderate and severe acute respiratory distress syndrome: a randomized controlled trial. JAMA. 2009;302(18):1977-84. https ://doi.org/10.1001/jama.2009.1614.

36. Ryan DW, Pelosi P. The prone position in acute respiratory distress syndrome. BMJ. 1996;312(7035):860-1. https://doi.org/10.1136/ bmj.312.7035.860.

37. Girard R, Baboi L, Ayzac L, et al. The impact of patient positioning on pressure ulcers in patients with severe ARDS: results from a multicentre randomised controlled trial on prone positioning. Intensive Care Med. 2014;40(3):397-403. https://doi.org/10.1007/ s00134-013-3188-1.

38. Moore Z, Patton D, Avsar P. Prevention of pressure ulcers among individuals cared for in the prone position: lessons for the COVID-19 emergency. J Wound Care. 2020;29(6):312-20. https ://doi.org/10.12968/jowc.2020.29.6.312.

39. Panchabhai TS, Bandyopadhyay D, Kapoor A, et al. Acute ischemic optic neuropathy with extended prone position ventilation in a lung transplant recipient. Int J Crit Illn Inj Sci. 2016;6(1):45-7. https://doi.org/10.4103/2229-5151.177367. 